\title{
Validation of a volume-averaged fiber-scale model for the oxidation of a carbon-fiber preform
}

\author{
Jean Lachaud * \\ UARC/UC Santa Cruz, Moffett Field, CA \\ Nagi N. Mansour ${ }^{\dagger}$ \\ NASA Ames Research Center, Moffett Field, CA \\ Alejandro Ceballos, Dušan Pejaković, Luning Zhang, and Jochen Marschall $\ddagger$ \\ SRI International, Menlo Park, CA
}

\begin{abstract}
The oxidation of Fiberform, an industrial carbon-fiber preform, has been studied in an oxidation reactor. The microscopic oxidation behavior of the fibers has been analyzed by scanning electron microscopy. The carbon fibers ablate showing progressive reduction of their diameter. The overall material recession occurs when the fibers are consumed. A reaction/diffusion-convection competition is shown to drive the oxidation process and control the depth of oxidation. A fiber-scale model is proposed for the prediction of carbonfiber preform oxidation. A macroscopic model is derived by volume-averaging the microscopic model and a porous-medium formulation is used to model mass transport in the preform. The proposed model has been implemented in a Carbon Oxidation Analysis Code based on OpenFOAM (COACO). Using inverse analysis, it was possible to estimate the intrinsic fiber reactivity and then validate the model. The reactivity obtained is surprisingly high compared to literature data. This is explained by the fact that the carbon fibers contain some traces of calcium and potassium, which are known to be catalysts for oxidation. They progressively accumulate at the surface in the form of combustion residues.
\end{abstract}

\section{Nomenclature}

$D \quad$ Diffusion coefficient, $m^{2} \cdot s^{-1}$

$d_{p} \quad$ Mean pore diameter

$f \quad$ Rarefaction function

$J \quad$ Molar oxidation flux, $\mathrm{mol} \cdot \mathrm{m}^{-2} \cdot \mathrm{s}^{-1}$

$k_{f} \quad$ Reactivity constant, $m \cdot s^{-1}$

$n \quad$ Surface normal, $m$

$P e \quad$ Péclet number

$r_{f} \quad$ Fiber radius, $m$

$S \quad$ Surface function

$s_{f} \quad$ Fiber-preform specific surface, $m^{2} \cdot m^{-3}$

$V \quad$ Averaging volume, $m^{3}$

$v \quad$ Recession velocity, $m \cdot s^{-1}$

Subscripts

$0 \quad$ Initial value

diss Hydrodynamic dispersion

eff Effective value

$f \quad$ Fiber

*Associate Scientist, AIAA senior member.

${ }^{\dagger}$ Chief Scientist for Modeling and Simulation, Space Technology Division. AIAA Associate fellow.

¥Senior Scientist, AIAA senior member.

Copyright (C) 2011 by the American Institute of Aeronautics and Astronautics, Inc. The U.S. Government has a royalty-free license to exercise all rights under the copyright claimed herein for Governmental purposes. All other rights are reserved by the copyright owner. 
$g \quad$ Gas phase

Conventions

$\partial_{t} \quad$ Time derivative, $s^{-1}$

$\partial_{\mathbf{x}} \quad$ Spatial derivative, $s^{-1}$

Symbols

$\epsilon \quad$ Volume fraction

$\eta \quad$ Tortuosity

$\Omega \quad$ Solid molar volume, $\mathrm{m}^{3} \cdot \mathrm{mol}^{-1}$

\section{Introduction}

No (known) material is able to withstand the severe hypersonic aerothermal environment encountered by space vehicles during high-speed atmospheric entry. High performance composites are used as thermal protection systems but they are progressively ablated by oxidation and sublimation. For very high speed entries, a new class of low-density ablative material has been introduced and validated in flight by the Stardust mission. They are obtained by impregnation of a low-density carbon-fiber preform with an expanded phenolic resin. The underlying idea is to fabricate a light, pyrolysing, ablating, and insulating material, which will still possess reasonable mechanical properties thanks to the carbon-fiber preform. Different carbon preforms may be used and ideally the quantity and properties of the polymer used for the impregnation could be adapted to the requirement of a given mission. We are interested in the oxidative behavior of bare carbon preforms for three reasons:

- The community might be interested in trying versions of low-density carbon/phenolic with small amount of phenolic, or even a non-impregnated carbon preform;

- Stardust post-flight analyses ${ }^{1}$ and theoretical analyses ${ }^{2,3}$ strongly suggest that subsurface ablation phenomena may occur (sublimation, oxidation, erosion) leading to the removal of the more reactive (or fragile) phenolic matrix leaving the carbon fiber unprotected under the surface;

- In a previous work, a multi-scale modeling approach for the finite-rate ablation of low density carbon/phenolic composites was proposed. ${ }^{3}$ Further investigations and numerous specific experiments are still needed to finish the development of this model and validate it. Validating a multiscale model for the carbon preform in absence of phenolic would be a first and encouraging step towards the completion of the general model.

We chose to study the oxidation of Fiberform, an industrial carbon preform manufactured by Fiber Material Inc, at a moderate temperature $(898 \mathrm{~K})$ and pressure $(0.13 \mathrm{~atm})$. The carbon Fiberform insulation is made of carbon fibers, with an aspect ratio (length to diameter) of about 100, bonded together with a small amount of phenolic resin and carbonized. ${ }^{4}$ The specimens tested in this study had an initial density of about $180 \mathrm{~kg} \cdot \mathrm{m}^{-3}$ and an initial porosity of about $90 \%$.

The objectives are the following:

- Test and analyze the oxidation behavior of Fiberform (section two);

- Develop a multi-scale model for carbon-fiber preforms and implement it in a simulation tool (section three);

- Compare experimental and theoretical results, estimate the effective reactivity of the carbon fibers, and assess the validity of the approach (section four).

In the conclusion section, we will summarize the results and briefly describe future plans.

\section{Experimental analysis}

In this section, we will describe the oxidation reactor used for the experiment and analyze, at the fiber scale, the behavior of Fiberform during oxidation using Scanning Electron Microscopy (SEM). 


\section{II.A. Description of the oxidation reactor}

A sketch of the reactor is presented in Fig. 1. The reactor consists of a $2.2 \mathrm{~cm}$ diameter quartz tube heated by a single-zone tube furnace capable of reaching temperatures up to $1500 \mathrm{~K}$. Regulated gas flows are introduced upstream of the furnace through electronic mass flow controllers and are exhausted downstream of the furnace by a roots-blower vacuum pumping system. For a given mass flow rate, reactor pressures can be adjusted using a throttle valve in line with the pumping manifold. The porous carbon plug is positioned snugly within the quartz tube near the center of the furnace. The absolute pressure upstream of the furnace $\left(p_{1}\right)$ and the differential pressure across the carbon plug $\left(p_{2}-p_{1}\right)$ are measured by several capacitance manometers. The downstream gas composition can be monitored by a residual gas analyzer.

Cylindrical Fiberform plugs of different lengths $(2$ to $5 \mathrm{~cm}$ ) were machined with their diameters slightly over $2.2 \mathrm{~cm}$ (by $\approx 0.2 \mathrm{~mm}$ ) so that they could be press-fit into the quartz tube. The cylindrical plug axis was coincident with the through-the-thickness direction of the Fiberform billet. Before and after oxidation testing, the length of each specimen was measured with $0.01 \mathrm{~mm}$ resolution using calipers and each specimen was weighed with $10^{-5} \mathrm{~g}$ resolution on a Mettler Toledo XP105 Analytical scale. Pretest and post-test specimen densities were computed from these sets of measurements. Oxidation tests were performed by heating the sample to the desired temperature under a flow of nitrogen (Matheson, Ultra High Purity), then switching to a flow of dry synthetic air (Matheson, Ultra Zero Purity) for a desired test time, and then switching back to nitrogen during the furnace cool down period.

Samples of various lengths between 2 and $5 \mathrm{~cm}$ were tested at a temperature of $898 \mathrm{~K}$. Similar results were obtained with all the samples. For the sake of clarity, we will focus the study on the analysis of a single specimen of initial length $2.03 \mathrm{~cm}$ and initial density $184 \mathrm{~kg} \cdot \mathrm{m}^{-3}$. The specimen was oxidized for 60 minutes under dry air at a mass flow rate of $2.149 \mathrm{mg} \cdot \mathrm{s}^{-1}$. The inlet pressure was $12.8 \mathrm{kPa}$ and the pressure difference across the sample was small compared to the static pressure ( $\Delta p=0.7 \mathrm{kPa}$ ). The bulk convective gas velocity approaching the sample was then $0.11 \mathrm{~m} \cdot \mathrm{s}^{-1}$. After the 60 minute oxidation test, the length of the sample was reduced to $1.33 \mathrm{~cm}$ (reduction of $0.7 \mathrm{~cm}$ ) and its density decreased to $177 \mathrm{~kg} \cdot \mathrm{m}^{-3}$ (i.e. by about $4 \%$ ).

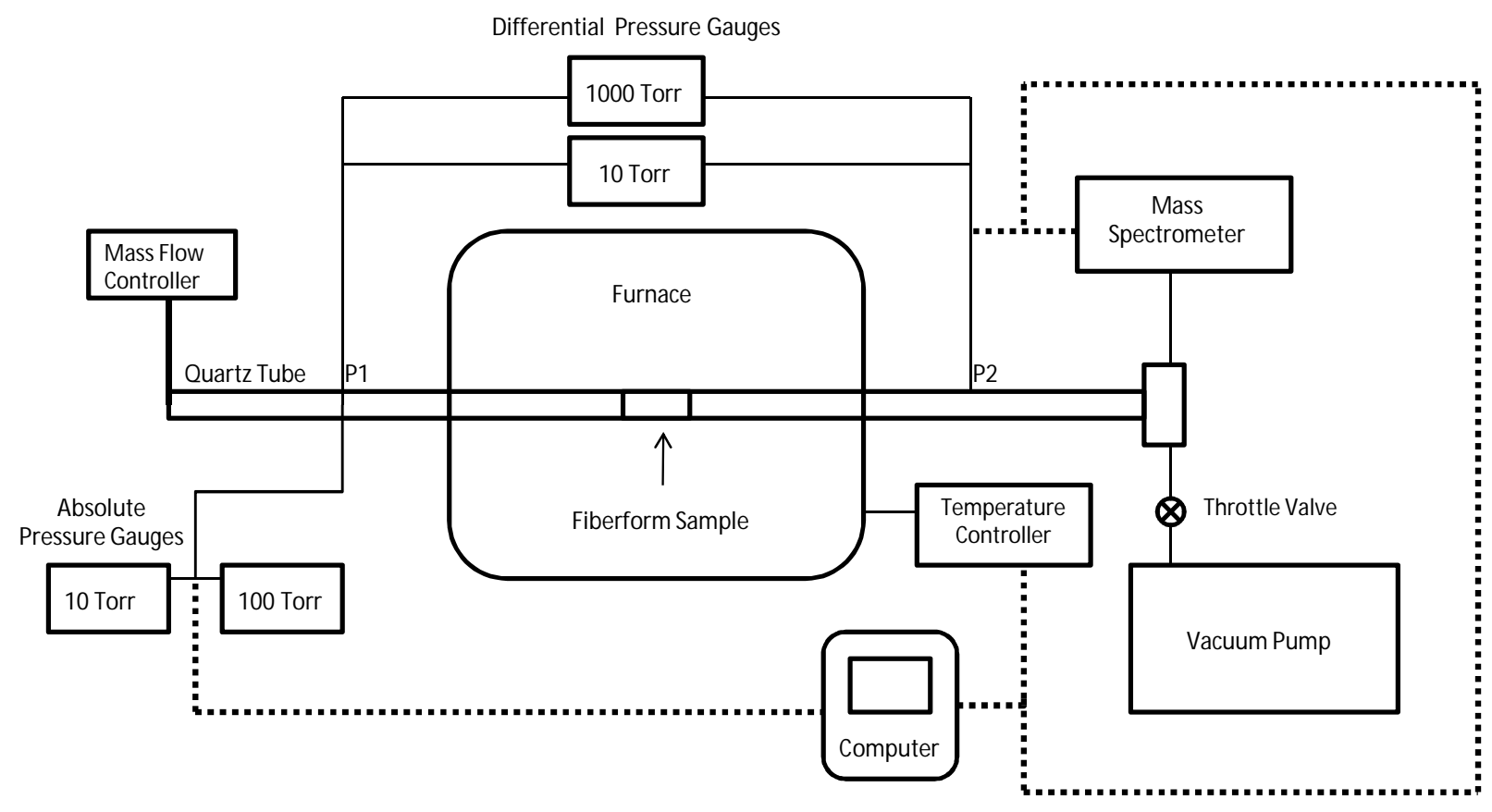

Figure 1. Diagram of the oxidation reactor. 


\section{II.B. Scanning electron microscopy analysis}

The small change in density and the significant recession of the sample is in agreement with the microscopic observations. As seen in Fig. 2 (flow is coming from the right), only the fibers beneath the surface facing into the air flow are significantly oxidized. This is characteristic of a diffusion-convection limited regime, where reaction mechanisms are faster than mass transport at the scale of interest. However, looking more closely to the Scanning Electron Micrograph on the right-hand side, we clearly see the evolution of the fiber diameter, as the fiber is being oxidized. This is the phenomenon that will need to be modeled together with the mass transport and the oxygen consumption by the fibers. Fig. 3 presents a comparison of the micro-structure in the non-oxidized zone far from the surface exposed to the air flow (left) and in the zone being oxidized (right). We see that the initial diameter of the fiber is about $10 \mu m$ and that it decreases with oxidation. We can also see clearly, on the non-oxidized material, the former phenolic resin from the fabrication process of Fiberform bonding the fibers at some of their intersections. During the manufacturing process of Fiberform, the phenolic polymer is fully carbonized at high temperature, leaving a carbon bond between the fibers. In the modeling section, we will assume that these bonds are homogeneously wrapped around the fibers. We can see here that this is not strictly true. We will also assume that the fibers are homogeneously oxidized, which may be generally valid but is obviously not locally correct as seen from the pitting features in Fig. 3 and from the irregularities in the thinning of the fiber in Fig. 2.

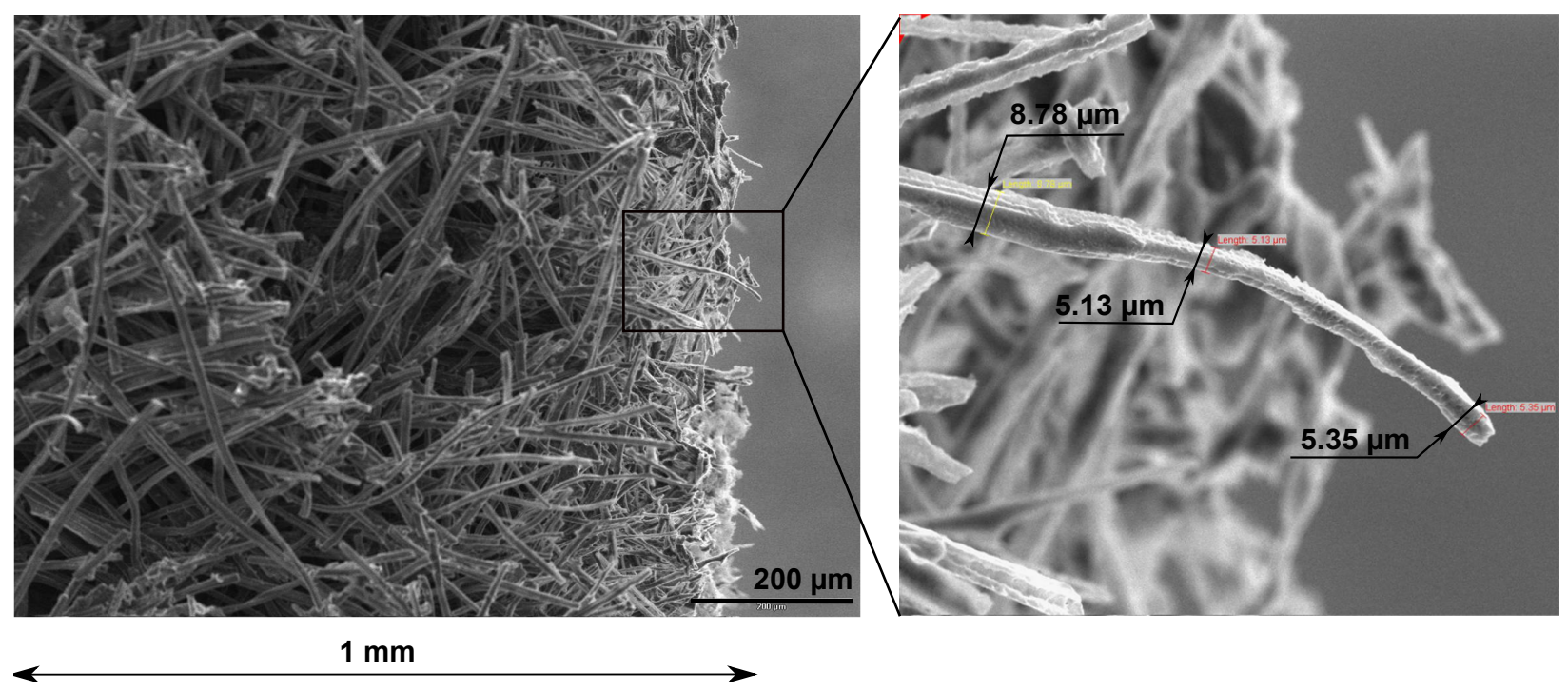

Figure 2. Scanning electron micrographs of the exposed side of the fiberform cylinder.

\section{Modeling and simulation}

In this section, we will present a model for the oxidation of Fiberform based on a multi-scale approach and we will describe the simulation tool implemented to test the model.

\section{III.A. Volume-averaged fiber-scale model}

The heterogeneous oxidation reaction in which carbon is consumed leads to the recession of the surface of the fibers (as seen in Fig. 3). The local motion of the fiber/fluid interface can be interpreted as a receding front. The interface is represented by a surface function $S(x, y, z, t)$ first-order differentiable almost everywhere, with a constant value (zero) at the interface. ${ }^{5}$ The function $S$ satisfies the differential equation ${ }^{6}$

$$
\partial_{t} S+\mathbf{v} \cdot \partial_{\mathbf{x}} S=0
$$

with the recession velocity, $\mathbf{v}$, modeled as 

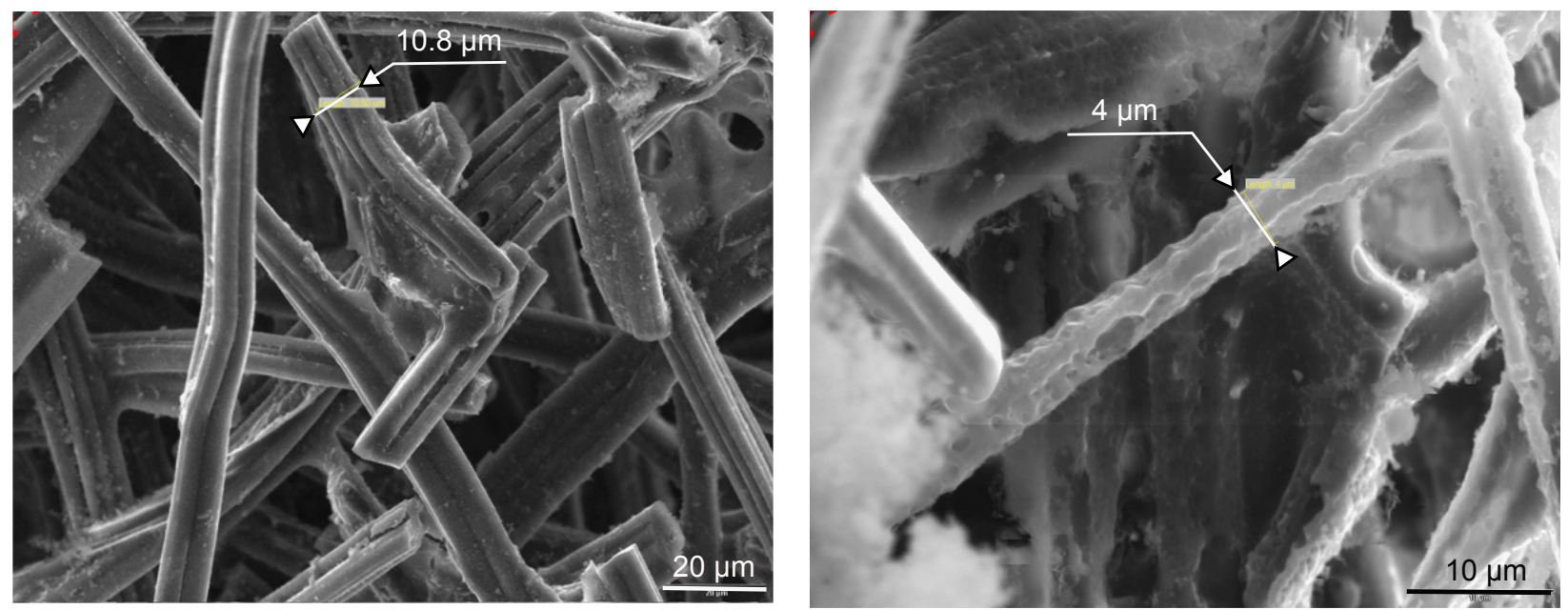

Figure 3. Comparison of the micro-structure in the non-oxidized zone far from the surface exposed to the air flow (left) and of the zone being oxidized (right).

$$
\mathbf{v}=\Omega J \mathbf{n}
$$

where $\Omega$ is the solid molar volume of the carbon fibers, $J$ is the molar oxidation flux, and $\mathbf{n}=\partial_{\mathbf{x}} S /\left\|\partial_{\mathbf{x}} S\right\|$ is the unit normal pointing outwards from the surface. In air at $898 \mathrm{~K}$, the chemical balance equation for the oxidation of solid carbon is

$$
\mathrm{C}_{\mathrm{s}}+\mathrm{O}_{2} \rightleftharpoons \mathrm{CO}_{2}
$$

Mass spectroscopy measurements at the outlet of the reactor have confirmed that carbon dioxide is the product of the reaction. We shall model the oxidation of carbon fibers in air at $898 \mathrm{~K}$ with a first order heterogeneous reaction, ${ }^{7}$ for which the local impinging molar flux density (on a fiber or carbonized matrix elementary surface) is given by

$$
J=k_{f} X
$$

where $X=X(x, y, z, t)$ is the oxygen concentration and $k_{f}$ is the fiber intrinsic reactivity. The carbon fibers are assumed homogeneous and isotropic at the microscopic scale.

For practical reasons, it is convenient to use several surface functions to describe distinct objects. For example, a surface function per fiber may be used to model Fiberform at the microscopic scale. The surface function equations may be solved by direct numerical simulation using high-resolution three-dimensional front-tracking algorithms at the fiber scale. ${ }^{8}$ An understanding of the oxidation process taking place at the fiber scale has been obtained thanks to direct numerical simulation presented in a previous work. ${ }^{2}$ However, direct numerical simulation is computationally expensive and not appropriate for design. Alternatively, when the surface function at the initial time (i.e. the initial geometry of the fibers) and the recession velocity $(\mathbf{v})$ are not too complex, analytical solutions may be obtained.

The fiber preform will be modeled as a random array of carbon cylinders. This material model is in qualitative agreement with the microscopic architecture seen in Fig. 2. The fiber length is large compared to its diameter (aspect ratio of about 100). Therefore, fibers are going to lose mass mainly due to radial recession; recession in length can be neglected. At the initial time, each fiber,$i$, can be modeled as an infinite cylinder of circular section, with a time-dependent geometry that may be defined in cylindrical coordinates $\left(e_{r_{i}}, e_{\theta_{i}}, e_{z_{i}}\right)$ in the frame of the fiber axis. Assuming that the fiber section remains circular during the oxidation process, one can remove the angular dependency, and the surface function may be written as $S_{i}\left(r_{i}, \theta_{i}, z_{i}, t\right)=r_{i}-r_{f}\left(z_{i}, t\right)$, which takes the value of 0 at the interface. The partial differential equation describing the evolution of the fiber radius $r_{f}\left(z_{i}, t\right)$ as a function of space and time is obtained by substituting this surface function in Eq. 1. After combining the expression for the fiber surface with Eqs. 2 and 4, we obtain

$$
\partial_{t} r_{f}\left(z_{i}, t\right)=-\Omega k_{f} X\left(z_{i}, t\right) \sqrt{1+\left(\frac{\partial r_{f}}{\partial z_{i}}\right)^{2}} \quad \text { for } \quad r_{f}>0
$$


Using this equation, we could compute the recession of each fiber as a function of its location, orientation and of time, but this would yield more information than we need at the macroscopic scale. For design purposes, it is sufficient to determine the average fiber diameter associated with an averaging volume, $\mathrm{V}(\mathrm{x}, \mathrm{y}, \mathrm{z})$, large compared with the fiber diameter but small compared with gradients at the macroscopic scale. In the averaging volume, we shall assume that the oxygen concentration is homogeneous. Under this assumption, the spatial derivative of the right hand side of Eq. 5 is of second order and will be neglected. Eq. 5 may then be rewritten in global coordinates as

$$
\partial_{t} r_{f}(x, y, z, t)=-\Omega k_{f}\langle X(x, y, z, t)\rangle^{g} \quad \text { for } \quad r_{f}>0
$$

where

$$
\langle X(x, y, z, t)\rangle^{g}=\frac{1}{V_{g}} \int_{V_{g}} X(x, y, z, t) d V
$$

with $V_{g}$ the volume of the gas phase contained in the averaging volume $V$. Eq. 6 expresses the intuitive fact that the local recession velocity of the fiber radius is proportional to the molar volume of the material, the reaction coefficient, and the local concentration of oxygen.

The (average) fiber volume fraction can be expressed as a function of the time dependent (average) fiber radius

$$
\epsilon_{f}=\epsilon_{f, 0}\left(\frac{r_{f}}{r_{f, 0}}\right)^{2}
$$

The effective surface of the Fiberform plug is at the location where the fiber volume fraction tends to zero, which occurs when the fiber radius tends to zero.

To solve Eq. 5, we would need to compute the local oxygen concentration everywhere in the porous medium. However, as previously mentioned, for design purposes, it is sufficient to solve Eq. 6 and therefore to determine the average oxygen concentration and the average rate of reaction.

Accounting for the volume sink term due to the heterogeneous consumption of oxygen, the averaged mass-balance equation in the porous medium closes as ${ }^{9}$

$$
\partial_{t}\left(\epsilon\langle X\rangle^{g}\right)+\partial_{\mathbf{x}} \cdot\left(-\left(D_{\text {eff }}+D_{\text {dis }}\right) \partial_{\mathbf{x}}\langle X\rangle^{g}\right)+f\left\langle\mathbf{v}_{\mathbf{g}}\right\rangle^{g} \cdot \partial_{\mathbf{x}}\langle X\rangle^{g}=-\langle X\rangle^{g} s_{f} k_{f}
$$

where $D_{e f f}, D_{d i s}, f$, and $\left\langle v_{g}\right\rangle^{g}$ are, respectively, the effective diffusion coefficient, a hydrodynamic dispersion coefficient, a rarefaction function, and the average velocity of the gas phase in the porous medium. The specific surface of the fiber preform $\left(s_{f}\right)$ treated as a random distribution of cylinders with a large aspect ratio, is given by

$$
s_{f}=r_{f} \frac{2 \epsilon_{f, 0}}{r_{f, 0}^{2}}
$$

For the conditions of the experiment, the (molecular) mean free path is $2.2 \mu \mathrm{m}$. The mean pore diameter is about $50 \mu \mathrm{m}$ for the Fiberform. The Knudsen number (ratio of the mean free path to the mean pore diameter) is then about 0.044. The flow in the porous medium is in the intermediate regime. In isotropic porous media, the volume-averaged diffusion (Fick) law keeps the same form in all regimes (continuum to Knudsen), but the effective diffusion coefficient becomes ${ }^{10}$

$$
D_{e f f}=\frac{\epsilon}{\eta} D_{r e f}
$$

where $D_{\text {ref }}$ is a reference diffusivity, corresponding to the longitudinal diffusivity into a capillary of diameter $d_{p}$. According to Bosanquet relation, ${ }^{10}$ close to the continuum limit we can assume that the reference diffusivity is equal to the bulk diffusivity. The tortuosity, $\eta$, is a geometric factor that characterizes the difference between a straight capillary and the actual tortuous medium for the molecule trajectories. The tortuosity of random fibrous media has been studied in a previous work. ${ }^{3}$ For random fibrous media with a porosity of 0.9 , the value of the tortuosity coefficient ranges from 1.1 in continuum regime to 2 in Knudsen regime. The tortuosity coefficient is found to be equal to 1.15 in the present conditions. ${ }^{3}$ The effective diffusion coefficient of oxygen in the Fiberform is estimated to be $7.6 \cdot 10^{-4} \mathrm{~m}^{2} \cdot \mathrm{s}^{-1}$ in the conditions of the 
experiment $(T=898 \mathrm{~K}, p=12.8 \mathrm{kPa})$. According to the averaging theory, the dispersion $\left(D_{\text {dis }}\right)$ is negligible for Péclet numbers smaller than unity. ${ }^{9}$ In porous media, the Péclet number is defined as ${ }^{9}$

$$
P e=\frac{\left\langle v_{g}\right\rangle^{g} d_{p}}{D_{b u l k}}\left(\frac{\epsilon}{1-\epsilon}\right)
$$

and is found to be around 0.05 in the present case. The rarefaction function, $f$, is taken equal to one since the regime is not rarefied. ${ }^{9}$

\section{III.B. Simulation tool: COACO-1.0}

The model presented in the previous section has been implemented in the framework of OpenFOAM, an open source computational fluid dynamic software (www.openfoam.com). A Carbon Oxidation Analysis Toolbox based on OpenFOAM [COACO] is being developed to analyze oxidation experiments, extract intrinsic oxidation rates, and test new macroscopic models. The first version of the solver, COACO-1.0, is an implementation of Eq. 7 and 9 using a first-order time-accurate finite-volume method, with implicit treatment of the diffusion, convection, and sink terms.

A more detailed description will be provided in the final version of the article.

\section{Numerical analysis}

In this section, we will reproduce the oxidation test, estimate the intrinsic reactivity of the fibers, and analyze and comment on the results.

\section{IV.A. Simulation results}

The diameter of the quartz reactor tube is very large compared to the fiber diameter and the gas flow in the porous medium is driven by pressure gradients. The size of boundary layer developing on the wall of the tube is then small. We may treat the mass transport in the tube as a one-dimensional problem. It is not necessary to model the entire inlet tube since we are interested in the oxidation of the sample only. However, the boundary condition is imposed at a distance from the surface for which the Péclet number becomes larger than one as an oxygen boundary layer forms at the surface of the sample (as seen in Fig. 4). The sample is a plug of $2.03 \mathrm{~cm}$ that has been oxidized for 1 hour at $898 \mathrm{~K}$ under an air pressure of $12800 \mathrm{~Pa}$. The velocity of the air flow was $v_{g}=0.11 \mathrm{~m} \cdot \mathrm{s}^{-1}$. In Fig. 4, the fiber diameter distribution is shown (on the left-hand side) for 4 different times, showing the reduction of the fiber diameter as the sample is oxidized. On this figure, the flow is coming from the right-hand side. A stagnation-line boundary layer forms at the surface of the sample. The oxygen concentration in the sample drops rapidly as oxygen is consumed by the fibers. A reaction/diffusion-convection competition is observed, resulting in the oxidation of the sample within the first few millimeters under the overall surface. This fact is in accordance with the mass spectroscopy measurements that show a full conversion of oxygen into carbon dioxide downstream of the specimen. At the end of the experiment, the total recession of the sample was $0.7 \mathrm{~mm}$. This data was used to estimate the intrinsic reactivity of the fibers. The intrinsic reactivity of the fibers in these conditions, $k_{f}$, is estimated to be around $1 \cdot 10^{-2} \mathrm{~m} \cdot \mathrm{s}^{-1}$. The fiber radius evolution under the surface displayed in Fig. 4 is in qualitative agreement with the SEM observations. In the final version of the article, we will present a quantitative comparison. We are planning to measure the density of the oxidized sample via a non-destructive X-ray technique and compare it to the numerical simulation results. This will help to refine the estimation of the intrinsic reactivity and provide a measure of uncertainty for the value obtained by inverse analysis.

\section{IV.B. Comments and open questions}

The value of the intrinsic reactivity of the carbon fibers found is surprisingly high compared to literature data. The reactivity of carbon fibers manufactured from the same precursor has been measured to be about $1 \cdot 10^{-4} \mathrm{~m} \cdot \mathrm{s}^{-1}$ using another experimental configuration. ${ }^{7}$ As discussed in a previous work, ${ }^{7}$ the reactivity of carbon fibers is known for being strongly influenced by the manufacturing process and by catalysts. Therefore, the possible presence of catalysts has been studied. 


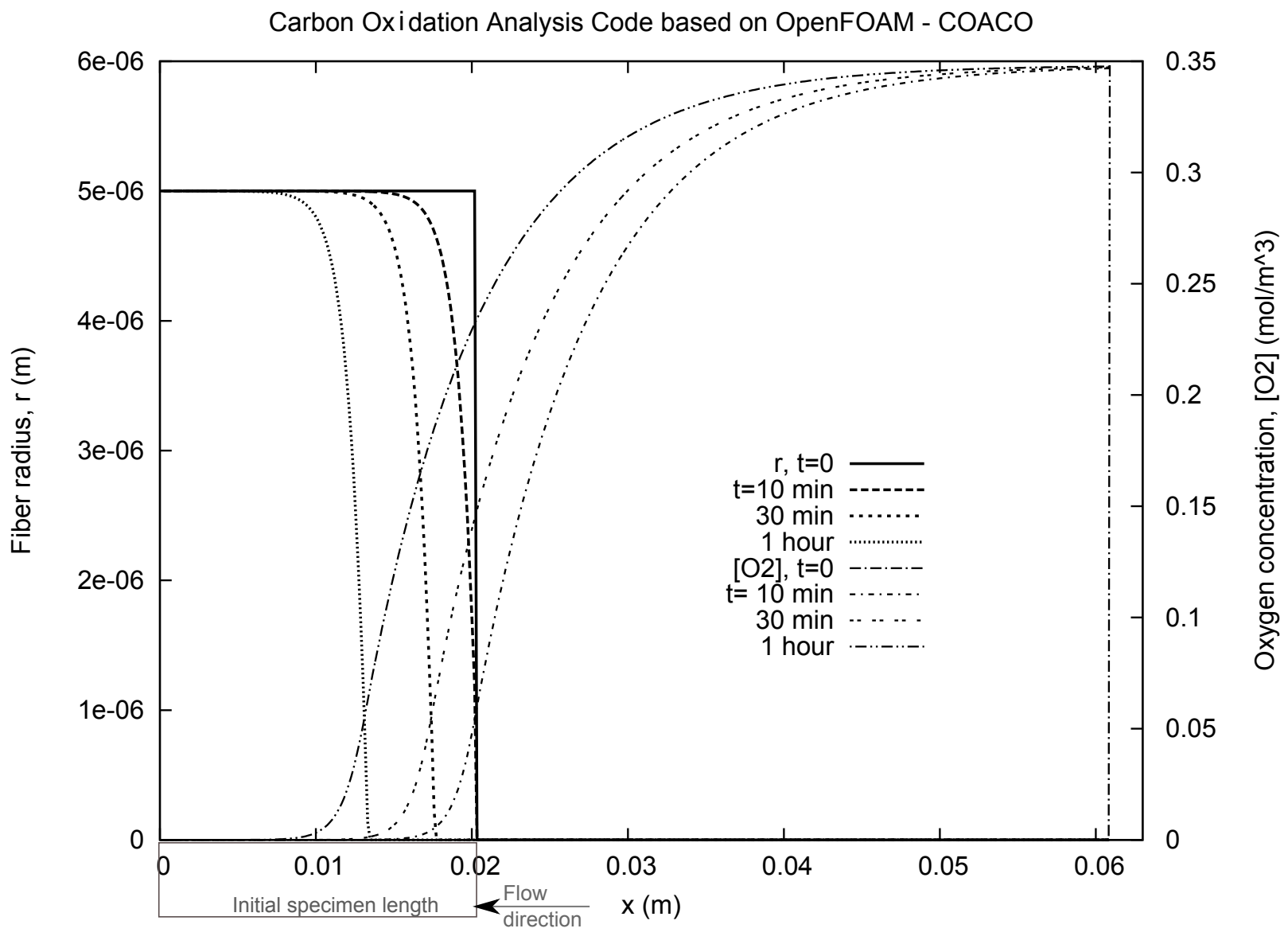

Figure 4. Time-dependent simulation of the fiber radius, sample recession, and oxygen concentration in the reactor.

Energy-Dispersive X-ray spectrometer (EDX) was used to analyze the elemental composition of the sample before and after oxidation. A beam of electrons is focused on the zone to be analyzed. The electronic excitation generates an X-ray response that is characteristic of the atomic composition. The non-oxidized carbon fibers contain traces of calcium that progressively accumulate during the combustion of the fibers to form clusters of ashes (combustion residues), as seen in the micrograph of Fig. 5 (in white). The EDX analysis suggests that the combustion residue is calcium carbonate $\left(\mathrm{CaCO}_{3}\right)$ with traces of silicon, sulfur, and potassium. Since no oxygen has been found in the original carbon fibers, calcium carbonate may form in the conditions of the experiment. Calcium and potassium have been shown to catalyze the oxidation of carbon in presence of air ${ }^{11}$ explaining the difference with literature data. Indeed, in the work previously mentioned, ${ }^{7}$ the carbon fibers had been treated at high temperature to remove the catalysts.

This observation may explains why calcium spectra had been observed by emission spectroscopy in the trail of Stardust during its re-entry. This will be developed further in the final version of the paper.

The open question that will also be developed in the final version of the paper is: should we treat Fiberform at high temperature to remove possible catalysts and improve its low temperature oxidation properties? For high speed re-entries this would be beneficial outside the diffusion-limited plateau, which is a function of the re-entry conditions (thickness of the boundary layer and density of the gas). Therefore, this question should be addressed when the re-entry parameters have been decided to optimize, if necessary, the material properties in the oxidation limited domain. 

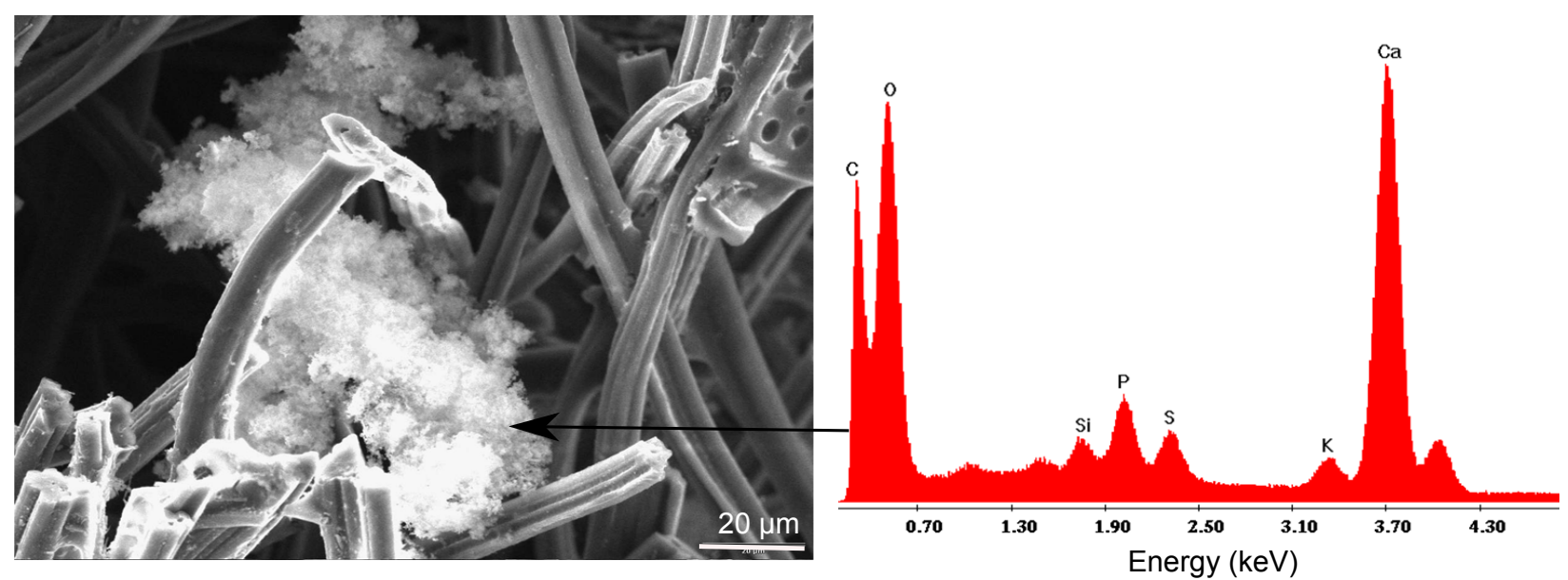

Figure 5. SEM-EDX analysis (arbitrary units) of the combustion residues showing the presence of carbon, oxygen, silicon, sulfur, potassium, and calcium.

\section{Conclusion}

The oxidation mechanism of a carbon-fiber preform under air has been studied and understood. The oxidation of the carbon fibers leads to a progressive reduction of their diameter. Theoretical derivations show that the velocity of the radius reduction is, as intuitively understood, proportional to the reaction rate, the local concentration of the oxidant, and the molar volume of the solid. Using a porous-medium formalism for mass transport the oxidation behavior of the preform may then be modeled using a physicsbased approach. A Carbon Oxidation Analysis Code based on OpenFOAM (COACO) has been implemented to test this model. The simulation results reproduce the qualitative experimental observations correctly. The intrinsic oxidation rate of the carbon fibers has been extracted by inverse analysis using the (quantitative) experimental recession data. The oxidation rate obtained is high compared to literature data. EnergyDispersive X-ray spectroscopy analyses have shown the presence of calcium and potassium in the fibers; these elements are known to be strong catalysts for the oxidation of carbon fibers. The presence of calcium in the fibers justifies the observation of calcium spectra in the trail of Stardust during its re-entry.

Further work will include a quantitative analysis of the effect of calcium on the oxidation rate of Fiberform and the pursuit of the study on impregnated ablative materials.

\section{Acknowledgments}

This research was partly supported by the hypersonics project of NASA's fundamental aeronautics program. The contributions of Alejandro Ceballos were supported by SRI's National Science Foundation Research for Undergraduates program. The authors would like to thank Jose Garcia-Chavez (NASA Ames) for helping with the SEM, Mairead Stackpoole (ERC/NASA Ames) for providing the Fiberform samples, and Ioana Cozmuta (ERC/NASA Ames) for very fruitful discussions.

\section{References}

\footnotetext{
${ }^{1}$ Stackpoole, M., Sepka, S., Cozmuta, I., and Kontinos, D., "Post-Flight Evaluation of Stardust Sample Return Capsule Forebody Heatshield Material," AIAA paper, Vol. 1202, January 2008, 12 p.

${ }^{2}$ Lachaud, J. and Mansour, N. N., "Microscopic scale simulation of the ablation of fibrous materials," Proc. $48^{\text {th }}$ AIAA Aerospace Sciences Meeting, Vol. 984, AIAA, 2010.

${ }^{3}$ Lachaud, J., Cozmuta, I., and Mansour, N. N., "Multiscale Approach to Ablation Modeling of Phenolic Impregnated Carbon Ablators," Journal of Spacecraft and Rockets, Vol. 47, No. 6, 2010, 12 pages.

${ }^{4}$ Tran, H. K., Johnson, C. E., Rasky, D. J., Hui, F. C. L., Hsu, M.-T., Chen, T., Chen, Y. K., Paragas, D., and Kobayashi, L., "Phenolic Impregnated Carbon Ablators (PICA) as Thermal Protection Systems for Discovery Missions," Tech. Rep. 110440, NASA Technical Memorandum, 1997.

${ }^{5}$ Katardjiev, I. V., Carter, G., Nobes, M. J., Berg, S., and Blom, H.-O., "Three-dimensional simulation of surface evolution during growth and erosion," Journal of Vacuum Science and Technology A, Vol. 12, No. 1, 1994, pp. 61-68.
} 
${ }^{6}$ Lachaud, J., Aspa, Y., and Vignoles, G. L., "Analytical modeling of the steady state ablation of a 3D C/C composite," International Journal of Heat and Mass Transfer, Vol. 51, No. 9-10, 2008, pp. 2614-2627, doi:10.1016/j.ijheatmasstransfer.2008.01.008.

${ }^{7}$ Lachaud, J., Bertrand, N., Vignoles, G. L., Bourget, G., Rebillat, F., and Weisbecker, P., "A theoretical/experimental approach to the intrinsic oxidation reactivities of C/C composites and of their components," Carbon, Vol. 45, No. 14, 2007, pp. 2768-2776, doi:10.1016/j.carbon.2007.09.034.

${ }^{8}$ Lachaud, J. and Vignoles, G. L., "A Brownian motion technique to simulate gasification and its application to C/C composite ablation," Computational Material Science, Vol. 44, No. 4, 2008, pp. 1034-1041, doi:10.1016/j.commatsci.2008.07.015.

${ }^{9}$ Whitaker, S., The method of volume averaging, Kluwer Academic Publisher, Dordrecht, The Netherlands, 1999.

${ }^{10}$ Tomadakis, M. M. and Sotirchos, S. V., "Ordinary, transition and Knudsen regime diffusion in random capillary structures," Chem. Eng. Sci., Vol. 48, No. 19, 1993, pp. 3323-3333.

${ }^{11} \mathrm{Wu}, \mathrm{X}$. and Radovic, L. R., "Catalytic oxidation of carbon/carbon composite materials in the presence of potassium and calcium acetates," Carbon, Vol. 43, 2005, pp. 333-344. 